\title{
Nonlocal relation between kinetic and exchange energy densities in the Bardeen model of a planar metal surface
}

\author{
N. H. March \\ Oxford University, Oxford, England; \\ Department of Physics, University of Antwerp (RUCA), Groenenborgerlaan, Antwerp, Belgium; \\ and The Abdus Salam International Centre for Theoretical Physics, Trieste, Italy
}

(Received 20 August 1999)

\begin{abstract}
The infinite barrier model of a planar metal surface, going back to Bardeen, is shown to permit the kinetic energy density to be written explicitly in terms of the ground-state electron density and its first derivative. The exchange energy density is then written in what proves to be an explicitly nonlocal form in terms of the electron density. Finally, by integrating the first-order equation for the electron density in terms of the kinetic energy density, a precise relation is established in this metal surface model between exchange and kinetic energy densities.
\end{abstract}

A long-term aim of density-functional theory has been to calculate directly from the ground-state density $\rho(\mathbf{r})$ the kinetic energy density $t(\mathbf{r})$. In this Brief Report, we achieve this aim in the admittedly very specialized example of the Bardeen model of a planar metal surface. ${ }^{1}$ This then prompts us to go on to explore the exchange energy density. In single-determinant models of many-electron systems, a class that embraces the Bardeen case considered throughout the present study, March and Santamaria ${ }^{2}$ noted that there was a fundamentally nonlocal relation between kinetic and exchange energies. Very recent work in this general area is that of Ernzerhof and Scuseria, ${ }^{3}$ where earlier references may be found.

In the present paper we first focus on density and kinetic energy. Immediately below, we merely quote the explicit expressions for the ground-state density $\rho(z)$ and the kinetic energy density $t(z)$, given, respectively, by Bardeen ${ }^{1}$ and by Moore and March. ${ }^{4}$ In the Bardeen model, electrons are confined by an infinite barrier in the $(x, y)$ plane, placed at $z$ $=0$. Then, if the Fermi wave number is $k$, Bardeen obtained the result

$$
\rho(z)=\rho_{0}\left[1-\frac{3 j_{1}(2 k z)}{2 k z}\right]
$$

where $\rho_{0}=k^{3} / 3 \pi^{2}$ and $j_{1}(x)=(\sin x-x \cos x) / x^{2}$ is the firstorder spherical Bessel function. Moore and $\mathrm{March}^{4}$ defined the kinetic energy density $t(\mathbf{r})$ from the idempotent firstorder density matrix $\gamma\left(\mathbf{r}, \mathbf{r}^{\prime}, k\right)$, which they derived for the Bardeen model through

$$
t(\mathbf{r})=-\left.\frac{\hbar^{2}}{2 m} \nabla_{r}^{2} \gamma\left(\mathbf{r}, \mathbf{r}^{\prime}, k\right)\right|_{\mathbf{r}^{\prime}=\mathbf{r}} .
$$

Since motion is free except along the $z$ axis in the model being employed here, $t(\mathbf{r}) \equiv t(z)$, and this is given in Eq. (6.12) of Ref. 4 as

$$
t(z)=\frac{\hbar^{2} k^{5}}{2 m \pi^{2}}\left[\frac{1}{5}-\frac{j_{1}(2 k z)}{2 k z}+\frac{2 j_{2}(2 k z)}{(2 k z)^{2}}\right]
$$

$j_{2}(x)$ entering Eq. (3), being the second-order spherical Bessel function. Just as we introduced the uniform density $\rho_{0}$ into Eq. (1), it will be convenient to express the MooreMarch result (3) in terms of the uniform electron-gas result for the kinetic energy per unit volume, $t_{0}$, say, given by

$$
t_{0}=c_{k} \rho_{0}^{5 / 3}, \quad c_{k}=\frac{3 h^{2}}{10 m}\left(\frac{3}{8 \pi}\right)^{2 / 3} .
$$

One then finds immediately

$$
\frac{t(z)-t_{0}}{t_{0}} \equiv \Delta t(z)=-\frac{5}{2 k z} j_{1}(2 k z)+\frac{10 j_{2}(2 k z)}{(2 k z)^{2}} .
$$

Defining similarly

$$
\Delta(z)=\frac{\rho(z)-\rho_{0}}{\rho_{0}},
$$

one has from Eq. (1) that

$$
-\frac{3 j_{1}(2 k z)}{2 k z}=\Delta(z)
$$

and this can be inserted into the first term on the right-hand side (RHS) of Eq. (5) to yield

$$
\Delta t(z)=\frac{5}{3} \Delta(z)+\frac{10 j_{2}(2 k z)}{(2 k z)^{2}} .
$$

Next we appeal to the mathematical identity

$$
\frac{d}{d x} \frac{j_{1}(x)}{x}=-\frac{j_{2}(x)}{x},
$$

which readily allows the last term in Eq. (8) to be reexpressed in terms of $\Delta(z)$ in Eq. (7). Hence the desired form of $\Delta t(z)$ is obtained solely in terms of the dimensionless "displaced" charge density by the infinite barrier as

$$
\Delta t(z)=\frac{5}{3} \Delta(z)+\frac{5}{6 k^{2} z} \frac{d \Delta(z)}{d z} .
$$


This has achieved the first objective of this Brief Report: namely, to express the kinetic energy density in terms of the ground-state density. We shall return to Eq. (10) below, after we have discussed the exchange energy density $\epsilon_{x}(\mathbf{r})$, defined in terms of the idempotent first-order density matrix $\gamma\left(\mathbf{r}, \mathbf{r}^{\prime}, k\right)$ introduced above through

$$
\epsilon_{x}(\mathbf{r})=-\frac{1}{4} e^{2} \int \frac{\gamma^{2}\left(\mathbf{r}, \mathbf{r}^{\prime}, k\right)}{\left|\mathbf{r}-\mathbf{r}^{\prime}\right|} d \mathbf{r}^{\prime} .
$$

Using the first-order density matrix of Ref. 4 in Eq. (11), Miglio, Tosi, and March ${ }^{5}$ have written $\epsilon_{x}(z)$ for the Bardeen model under discussion in the form

$$
\epsilon_{x}(z)=-\frac{3 e^{2} \rho_{0} k}{4 \pi} J(2 k z),
$$

where $J(2 k z)$ is written quite explicitly. Unfortunately, in the final form given for $J$ by Miglio, Tosi, and March, it has not proved possible, motivated by the above result, Eq. (10), for $\Delta t(z)$, to express $\epsilon_{x}(z)$ solely in terms of the displaced charge $\Delta(z)$. Therefore, we have returned to an earlier, integral form for $J(2 \mathrm{kz})$ given in Ref. 5, namely,

$$
J(2 k z)=\left(k^{2} / z\right) \int_{0}^{\infty} d R \int_{h(R, z)}^{R+2 z} d R^{\prime} R^{\prime}\left[\frac{j_{1}(k R)}{k R}-\frac{j_{1}\left(k R^{\prime}\right)}{k R^{\prime}}\right]^{2},
$$

where

$$
h(R, z)=\left\{\begin{array}{c}
2 z-R \text { for } R<z \\
R \text { for } R>z
\end{array}\right.
$$

But now one can use again in this integral expression for $J(2 k z)$ the result (7), to find

$$
\begin{aligned}
\epsilon_{x}(z)= & -\frac{3 e^{2} \rho_{0}}{4 \pi} \frac{k^{3}}{z} \int_{0}^{\infty} d R \int_{h(R, z)}^{R+2 z} d R^{\prime} R^{\prime} \\
& \times\left[-\frac{1}{3} \Delta\left(\frac{R}{2}\right)+\frac{1}{3} \Delta\left(\frac{R^{\prime}}{2}\right)\right]^{2},
\end{aligned}
$$

which is the result analogous to Eq. (10) for the kinetic energy density in this metal surface model.

However, to complete the aims of this investigation, we finally wish to express the exchange energy density $\epsilon_{x}(z)$ in terms of the kinetic energy density $t(z)$. Fortunately the sim- plicity of Eq. (10) relating $\Delta t(z)$ to the displaced charge $\Delta(z)$ enables us to solve for this latter quantity, characterizing $\epsilon_{x}(z)$ through Eq. (15). We first seek a solution of the RHS of Eq. (10) set equal to zero, when we find a result of the form $A \exp \left(-k^{2} s\right)$, with $s=z^{2}$. Thus we assume the generalization

$$
\Delta(s)=A(s) \exp \left(-k^{2} s\right)
$$

and substituting in Eq. (10) we obtain the function $A(s)$ as

$$
A(s)=\frac{3}{5} k^{2} \int_{l}^{s} \Delta t(q) \exp \left(k^{2} q\right) d q
$$

where the lower limit $l$ must be chosen to give $\Delta(z=0)=$ -1 . This has then achieved the final objective of this investigation, for having $\Delta(z)$ in terms of $\Delta t(z)$ through Eqs. (16) and (17), we can substitute for $\Delta(R / 2)$, etc., in Eq. (15) in favor of $\Delta t$.

In summary, the main results of this Brief Report are (1) Eq. (10), giving the kinetic energy density $t(z)$ in terms of the ground-state charge density plus its first derivative, (2) Eq. (15) for the exchange energy density $\epsilon_{x}(z)$, again in terms of the ground-state electron density, and (3) Eq. (16), giving the electron density as an explicit integral of the kinetic energy density, which then allows an explicit nonlocal expression to be written for $\epsilon_{x}(z)$ in terms of the kinetic energy density $t(z)$.

Two final comments are called for. First, within densityfunctional theory itself, refined treatments of the electron density $\rho(z)$ through a planar metal surface exist, which transcend the Bardeen result (1), though numerical procedures have to be invoked. ${ }^{6}$ Second, and in the same context, some progress has also been made in constructing a (partial) functional derivative of the exchange energy density in Eq. (11) to obtain an exchange potential ${ }^{7}$ within a jellium model framework, but further work remains to be done on this aspect of the problem.

The author wishes to thank Professor V. E. Van Doren for much support and for his continuing interest. This work was brought to fruition during a visit to the Abdus Salam International Centre for Theoretical Physics, Trieste, Italy. Thanks are due to Professor Yu Lu and his colleagues for a most stimulating environment and for generous hospitality.
${ }^{1}$ J. Bardeen, Phys. Rev. 49, 653 (1936).

${ }^{2}$ N. H. March and R. Santamaria, Int. J. Quantum Chem. 39, 585 (1991); see also A. Holas and N. H. March, Phys. Chem. Liq. 25, 43 (1992).

${ }^{3}$ M. Ernzerhof and G. E. Scuseria, J. Chem. Phys. 111, 911 (1999).

${ }^{4}$ I. D. Moore and N. H. March, Ann. Phys. (N.Y.) 97, 136 (1976).

${ }^{5}$ L. Miglio, M. P. Tosi, and N. H. March, Surf. Sci. 111, 119
(1981).

${ }^{6}$ See, for example, N. D. Lang in Theory of the Inhomogeneous Electron Gas, edited by S. Lundqvist and N. H. March (Plenum, New York, 1983), p. 309.

${ }^{7}$ A. Solomatin, V. Sahni, and N. H. March, Phys. Rev. B 49, 16856 (1994); see also A. Solomatin and V. Sahni, Ann. Phys. (N.Y.) 268, 149 (1998). 